\title{
OPTIMAL MULTIPLE-PASS AEROASSISTED PLANE CHANGE
}

\author{
NGUYEN X. VINH \\ Department of Aerospace Engineering, The University of Michigan, Ann Arbor, MI 48109-2140, U.S.A. \\ and \\ DER-Ming MA \\ Chung Shan Institute of Science and Technology, Lung-Tan, Taiwan 32526, Republic of China
}

(Received 31 August 1989; received for publication 19 September 1990)

\begin{abstract}
This paper presents the exact dimensionless equations of motion and the necessary conditions for the computation of the optimal trajectories of a hypervelocity vehicle flying through a non-rotating spherical planetary atmosphere. Numerical solution is then presented for the case when the vehicle makes several passages through the atmosphere near the perigee of its orbit. While the orbit is slowly contracting, aerodynamic maneuver is performed to obtain the maximum plane change. Several plots were presented to show the optimal variations of the lift coefficient and the bank angle and the various elements of the orbit.
\end{abstract}

\section{INTRODUCTION}

In recent years, there has been strong interest in the problem of optimal aeroassisted orbit transfer. A recent survey by Mease [1] has provided an extensive list of references. For the sake of generality Vinh et al. have advocated the use of dimensionless variables and in particular the so called Chapman's variable to write the equations of motion [2,3]. In this way, the formulation is free of the physical characteristics of the vehicle, except for the maximum lift-to-drag ratio, $E^{*}$, while the characteristic of the atmosphere is simply represented by a constant parameter $k^{2}=\beta r \quad(=900$ for the Earth). The only minor deficiency is that Chapman's altitude variable, $Z$, which is essentially the dimensionless density, can become negative during the integration if the altitude becomes very large. If the maneuver consists of one single passage through the atmosphere, then we can consider the point $Z \approx 0$ as the exit point. But for the case where, after reaching the apogee, the vehicle returns for another passage, to maintain the continuity for the numerical integration, it is necessary to retain the exponential nature of the atmospheric density.

In this paper, we first derive the exact dimensionless equations of motion and the necessary conditions for the computation of the optimal trajectories of a lifting hypervelocity vehicle flying through a non-rotating spherical planetary atmosphere to accommodate the case where the flight reaches the near vacuum. It will be shown that, in addition to the maximum lift-todrag, the characteristics of the vehicle include a certain ballistic factor, $B$. But, fortunately, just as in the case of the contraction of the orbit, this factor is insensitive to the relationship between the major axis and the eccentricity of the current orbit. Hence, in this new formulation, the generality of the results is preserved.

As an application, we present the numerical solution of the problem of optimal aeroassisted plane change by multiple passages through the Earth's atmosphere.

\section{EQUATIONS OF MOTION}

For the flight over a spherical, non-rotating planet of a non-thrusting, aerodynamic vehicle with the lift coefficient $C_{\mathrm{L}}$ and the drag coefficient $C_{\mathrm{D}}$, it is customary to use the equations of motion with the notation in Fig. 1. We have [2]

$$
\begin{aligned}
\frac{\mathrm{d} r}{\mathrm{~d} t} & =V \sin \gamma, \\
\frac{\mathrm{d} \theta}{\mathrm{d} t} & =\frac{V \cos \gamma \cos \psi}{r \cos \phi}, \\
\frac{\mathrm{d} \phi}{\mathrm{d} t} & =\frac{V \cos \gamma \sin \psi}{r}, \\
\frac{\mathrm{d} V}{\mathrm{~d} t} & =-\frac{\rho S C_{\mathrm{D}} V^{2}}{2 m}-g \sin \gamma, \\
V \frac{\mathrm{d} \gamma}{\mathrm{d} t} & =\frac{\rho S C_{\mathrm{L}} V^{2}}{2 m} \cos \sigma-\left(g-\frac{V^{2}}{r}\right) \cos \gamma, \\
V \frac{\mathrm{d} \psi}{\mathrm{d} t} & =\frac{\rho S C_{\mathrm{L}} V^{2}}{2 m \cos \gamma} \sin \sigma-\frac{\mathrm{V}^{2}}{r} \cos \gamma \cos \psi \tan \phi
\end{aligned}
$$

where the bank angle $\sigma$ is defined as the angle between the local vertical plane containing the velocity and the plane containing the velocity and the aerodynamic force. 


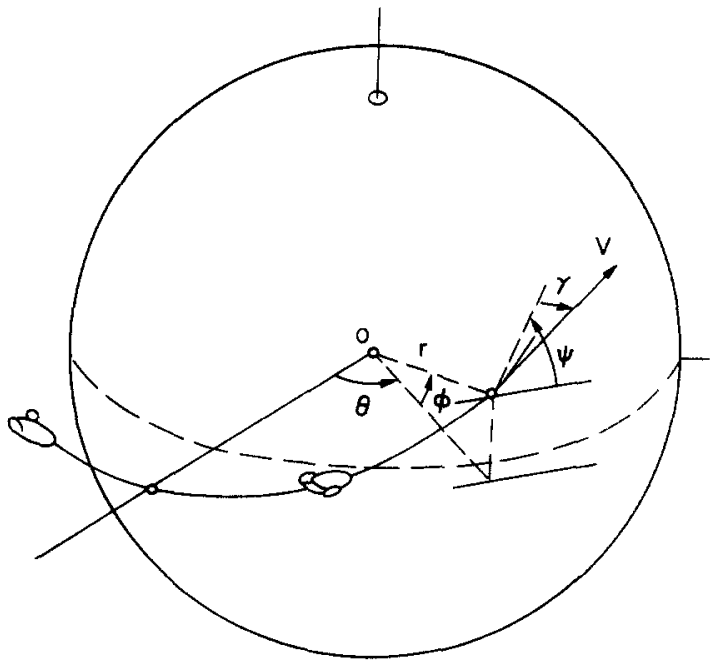

Fig. 1. Spherical coordinates for atmospheric flight.

For a smooth transition between atmospheric flight and flight in the vacuum, we define the dimensionless variables

$$
\begin{aligned}
& u=\frac{V^{2}}{g_{0} r_{0}}, \\
& h=\frac{r-r_{0}}{r_{0}}=\frac{y}{r_{0}} .
\end{aligned}
$$

to represent the speed and the altitude variables and the dimensionless arc length

$$
s=\int_{0}^{t} \frac{V}{r} \cos \gamma \mathrm{d} t
$$

to replace the time as the independent variable. The reference distance $r_{0}$ is the initial perigee distance. The drag polar used is the parabolic drag polar

$$
C_{\mathrm{D}}=C_{\mathrm{D}_{0}}+K C_{\mathrm{L}}^{2}
$$

with the condition at maximum lift-to-drag ratio

$$
\begin{aligned}
& C_{\mathrm{L}}=C_{\mathrm{L}}^{*}=\sqrt{C_{\mathrm{D}_{0}} / K}, \\
& C_{\mathrm{D}}=C_{\mathrm{D}}^{*}=2 C_{\mathrm{D}_{0}}, \\
& E^{*}=C_{\mathrm{L}}^{*} / C_{\mathrm{D}}^{*} .
\end{aligned}
$$

The aerodynamic control can be modeled as the modulation of the vertical and the lateral component of the normalized lift coefficient $\lambda$

$$
C=\lambda \cos \sigma, \quad S=\lambda \sin \sigma,
$$

where

$$
\lambda=\frac{C_{\mathrm{L}}}{C_{\mathrm{L}}^{*}} .
$$

Using a Newtonian gravitational field

$$
\frac{g}{g_{0}}=\frac{r_{0}^{2}}{r^{2}}
$$

and a locally exponential atmosphere

$$
\rho=\rho_{0} \exp (-\beta y)=\rho_{0} \exp \left(-\beta r_{0} \mathrm{~h}\right)=\rho_{0} \exp (-h / \epsilon)
$$

we obtain the universal equations for flight in both inside the atmosphere and in the vacuum:

$$
\begin{aligned}
\frac{\mathrm{d} h}{\mathrm{~d} s}= & (1+h) \tan \gamma, \\
\frac{\mathrm{d} \theta}{\mathrm{d} s}= & \frac{\cos \psi}{\cos \phi}, \\
\frac{\mathrm{d} \phi}{\mathrm{d} s}= & \sin \psi, \\
\frac{\mathrm{d} \psi}{\mathrm{d} s}= & \frac{B \lambda \sin \sigma(1+h) \exp (-h / \epsilon)}{\cos ^{2} \gamma} \\
& -\cos \psi \tan \phi, \\
\frac{\mathrm{d} u}{\mathrm{~d} s}= & -\frac{B(1+h) u\left(1+\lambda^{2}\right) \exp (-h / \epsilon)}{E^{*} \cos \gamma} \\
& -\frac{2}{(1+h)} \tan \gamma, \\
\frac{\mathrm{d} \gamma}{\mathrm{d} s}= & \frac{B(1+h) \lambda \cos \sigma \exp (-h / \epsilon)}{\cos \gamma} \\
& +1-\frac{1}{u(1+h)} .
\end{aligned}
$$

In the equations above, we have defined

$$
\epsilon=1 / \beta r_{0} \text { and } B=\frac{\rho_{0} S C_{\mathrm{L}}^{*} r_{0}}{2 m}
$$

In these equations the only physical characteristics of the vehicle are its maximum lift-to-drag ratio, $E^{*}$, and the ballistic coefficient $B$ specifying the starting flight altitude. We consider the case $E^{*}=1.5$ for the computation, and take a value $B$ low enough for the vehicle to stay in orbit for several revolutions. The value $1 / \epsilon=\beta r_{0}=900$ used is representative of the Earth's atmosphere.

\section{NECESSARY CONDITIONS FOR OPTIMALITY}

Introducing the adjoint variables $p_{x}$, we form the Hamiltonian

$$
\begin{aligned}
H= & p_{h}(1+h) \tan \gamma+p_{\theta} \frac{\cos \psi}{\cos \phi} \\
& +p_{\psi}\left[\frac{B \lambda \sin \sigma(1+\mathrm{h}) \exp (-h / \epsilon)}{\cos ^{2} \gamma}\right. \\
& -\cos \psi \tan \phi] \\
& -p_{u}\left[\frac{B(1+h) u\left(1+\lambda^{2}\right) \exp (-h / \epsilon)}{E^{*} \cos \gamma}\right. \\
& \left.+\frac{2}{(1+h)}\right]+p_{\phi} \sin \psi \\
& +p_{\gamma}\left[\frac{B(1+\mathrm{h}) \lambda \cos \sigma \exp (-h / \epsilon)}{\cos \gamma}\right. \\
& \left.+1-\frac{1}{u(1+h)}\right] .
\end{aligned}
$$


The maximization of the Hamiltonian with respect to the controls $\lambda$ and $\sigma$ leads to the optimal law

$$
C=\frac{E^{*} p_{\gamma}}{2 p_{u} u}, \quad S=\frac{E^{*} p_{\psi}}{2 p_{u} u \cos \gamma} .
$$

Along the optimal trajectory, the adjoint variable $p_{x}$, for any state variable $x$, satisfies the adjoint equation

$$
\frac{\mathrm{d} p_{x}}{\mathrm{~d} s}=-\frac{\partial H}{\partial x} .
$$

Explicitly, we have

$$
\begin{aligned}
\frac{\mathrm{d} p_{h}}{\mathrm{~d} s}= & -B \exp (-h / \epsilon)\left(1-\frac{1+h}{\epsilon}\right) \\
& \times\left[\frac{p_{\psi} \lambda \sin \sigma}{\cos ^{2} \gamma}-\frac{p_{u} u\left(1+\lambda^{2}\right)}{E^{*} \cos \gamma}+\frac{p_{\gamma} \lambda \cos \sigma}{\cos \gamma}\right] \\
& -p_{h} \tan \gamma-\frac{2 p_{u}}{(1+h)^{2}} \tan \gamma-\frac{p_{\gamma}}{u(1+h)^{2}}, \\
\frac{\mathrm{d} p_{\theta}}{\mathrm{d} s}= & 0, \\
\frac{\mathrm{d} p_{\psi}}{\mathrm{d} s}= & -p_{\phi} \cos \psi+\frac{\sin \psi}{\cos \phi}\left(-p_{\psi} \sin \phi+p_{\theta}\right), \\
\frac{\mathrm{d} p_{\phi}}{\mathrm{d} s}= & -\frac{\cos \psi}{\cos ^{2} \phi}\left(p_{0} \sin \phi-p_{\psi}\right), \\
\frac{\mathrm{d} p_{u}}{\mathrm{~d} s}= & \frac{p_{u} B(1+h)\left(1+\lambda^{2}\right) \exp (-h / \epsilon)}{E^{*} \cos \gamma}-\frac{p_{\gamma}}{u^{2}(1+h)} \\
\frac{\mathrm{d} p_{\gamma}}{\mathrm{d} s}= & -p_{h}(1+h) \sec ^{2} \gamma \\
& -\frac{2 p_{\psi} B \lambda \sin \sigma \sin \gamma(1+h) \exp (-h / \epsilon)}{\cos ^{3} \gamma} \\
& +\frac{p_{u} u B\left(1+\lambda^{2}\right) \sin \gamma(1+h) \exp (-h / \epsilon)}{E^{*} \cos ^{2} \gamma} \\
& -\frac{p_{\gamma} B \lambda \cos \sigma \sin \gamma(1+h) \exp (-h / \epsilon)}{\cos ^{2} \gamma} \\
& +2 p_{u} \frac{\sec c^{2} \gamma}{(1+h)} \cdot \\
&
\end{aligned}
$$

It is known that the problem has the following integrals [3]

$$
\begin{aligned}
& H=c_{0}, \\
& p_{\theta}=c_{1}, \\
& p_{\phi}=c_{2} \sin \theta-c_{3} \cos \theta, \\
& p_{\psi}=c_{1} \sin \phi+\left(c_{2} \cos \theta+c_{3} \sin \theta\right) \cos \phi,
\end{aligned}
$$

where the $c_{i}$ are constants of integration. In this problem of optimal plane change, we are concerned with speed depletion and heading angle without prescribing the final arc length $s_{\mathrm{f}}$ and final longitude $\theta_{\Gamma}$. Hence, by the transversality condition

$$
c_{0}=0, \quad c_{1}=0 .
$$

With the four integrals, only two of the remaining adjoint equations need to be integrated. The inte- gration requires guessing two initial values which, together with $c_{2}$ and $c_{3}$, constitute a four-parameter problem. By normalizing the adjoint variables, we are led to a three-parameter problem. The difficulty in guessing these parameters can be slightly alleviated by using the controls $C$ and $S$, as given in eqn (14), to replace the adjoint variables. By taking the derivatives of these equations, using the equations for the adjoint variables according to the optimal condition (16), we have directly the equations for the controls

$$
\begin{aligned}
\frac{\mathrm{d} C}{\mathrm{~d} s}= & \frac{1}{\cos ^{2} \gamma}\left[\frac{B(1+h) \exp (-h / \epsilon)}{2}\right. \\
& \left.\times\left(1-C^{2}-3 S^{2}\right) \sin \gamma+\frac{E^{*}}{u(1+h)}-F\right] \\
& +\frac{2 C}{E^{*} u(1+h)}\left[C+E^{*} \tan \gamma\right]
\end{aligned}
$$

and

$$
\begin{aligned}
\frac{\mathrm{d} S}{\mathrm{~d} s}= & -S \sin \psi \tan \phi+S \tan \gamma \\
& \times\left[1+\frac{1}{u(1+h)}+\frac{B(1+h) C \exp (-h / \epsilon)}{\cos \gamma}\right] \\
& -G \frac{\cos \psi}{\cos \gamma}+\frac{2 S C}{E^{*} u(1+h)}
\end{aligned}
$$

where $F$ and $G$ are defined as the ratios

$$
F=\frac{E^{*} p_{h}(1+h)}{2 u p_{u}}, \quad G=\frac{E^{*} p_{\phi}}{2 u p_{u}} .
$$

The equations for $F$ and $G$ can also be easily obtained as

$$
\begin{aligned}
\frac{\mathrm{d} F}{\mathrm{~d} s}= & \frac{B(1+h) \exp (-h / \epsilon)}{2 \cos \gamma}\left[1-\frac{(1+h)}{\epsilon}\right] \\
& \times\left(1-S^{2}-C^{2}\right) \\
& +\frac{2 F-E^{*}}{E^{*} u(1+h)}\left[C+E^{*} \tan \gamma\right]
\end{aligned}
$$

and

$$
\frac{\mathrm{d} G}{\mathrm{~d} s}=S \frac{\cos \psi \cos \gamma}{\cos ^{2} \phi}+\frac{2 G}{E^{*} u(1+h)}\left[C+E^{*} \tan \gamma\right] .
$$

In summary, besides the six state equations (11), we have four equations providing directly the controls $C$ and $S$ and the accessory variables $F$ and $G$. Their integration requires guessing three initial parameters $S_{0}, F_{0}$ and $G_{0}$ at the initial time, while $C_{0}$ can be computed from the Hamiltonian integral, with $c_{0}=c_{1}=0$ :

$$
\begin{aligned}
H= & \frac{2 u p_{u}}{E^{*}}\left[F \tan \gamma+G \sin \psi-\frac{B(1+h) \exp (-h / \epsilon)}{2 \cos \gamma}\right. \\
& \times\left(1-S^{2}-C^{2}\right)-S \cos \gamma \cos \psi \tan \phi \\
& \left.-\frac{E^{*} \tan \gamma}{u(1+h)}+C\left(1-\frac{1}{u(1+h)}\right)\right]=0
\end{aligned}
$$


Notice that we can use this integral and the integrals (17) to eliminate the equations for $F$ and $G$. From eqns (14) and (21), we have

$$
\frac{S \cos \gamma}{G}=\frac{p_{\psi}}{p_{\phi}} .
$$

With the integrals (17), we obtain

$$
\begin{aligned}
\frac{S \cos \gamma}{G} & =\frac{\left(c_{2} \cos \theta+c_{3} \sin \theta\right) \cos \phi}{c_{2} \sin \theta-c_{3} \cos \theta} \\
& =-\frac{\sin (\eta+\theta)}{\cos (\eta+\theta)} \cos \phi
\end{aligned}
$$

or

$$
S \cos \gamma+G \tan (\eta+\theta) \cos \phi=0
$$

where

$$
\eta=\tan ^{-1}\left(c_{2} / c_{3}\right)
$$

In practice, we integrate all four adjoint equations and use eqns (24) and (26) as verification of the accuracy of the numerical solution.

It is proposed for a prescribed speed depletion to maximize the plane change, $I$. Hence, we have the cost function to be minimized.

$$
J=\cos I_{\mathrm{f}}=\cos \phi_{\mathrm{f}} \cos \psi_{\mathrm{f}} .
$$

By the transversality conditions

$$
\begin{aligned}
& p_{\phi_{\mathrm{f}}}=-\frac{\partial J}{\partial \phi_{\mathrm{f}}}=\sin \phi_{\mathrm{f}} \cos \psi_{\mathrm{f}}, \\
& p_{\psi_{\mathrm{f}}}=-\frac{\partial J}{\partial \psi_{\mathrm{f}}}=\cos \phi_{\mathrm{f}} \sin \psi_{\mathrm{f}} .
\end{aligned}
$$

Then, at the final time

$$
\frac{S_{\mathrm{f}} \cos \gamma_{\mathrm{f}}}{G_{\mathrm{f}}}=\frac{P_{\psi_{\mathrm{f}}}}{P_{\phi_{\mathrm{f}}}}=\frac{\tan \psi_{\mathrm{f}}}{\tan \phi_{\mathrm{f}}}=-\tan \left(\eta+\theta_{\mathrm{f}}\right) \cos \phi_{\mathrm{f}} .
$$

Explicitly, the transversality condition to be satisfied at the final time is

$$
\tan \psi_{\mathrm{f}}+\sin \phi_{\mathrm{f}} \tan \left(\eta+\theta_{\mathrm{f}}\right)=0 .
$$

\section{EXACT SOLUTIONS FOR OPTIMAL PLANE CHANGE}

The difficulty in solving the optimal control problem in three dimensions with lift and bank control is in the sensitivity of the initial values of the adjoint variables. The initial value of any arbitrary adjoint variable may vary widely. Unless the initial adjoint values are not sensitive to small changes in the final values, a problem can be extremely difficult to solve.

The four parameters $C, S, F$ and $G$ defined in Section 3 can partially alleviate this problem. $S$ and $C$ are physical variables: they represent the lateral and vertical components of the normalized lift coefficient, respectively. Hence, the initial values of these two variables can be estimated from physical knowledge, such as the fact that an optimal trajectory is likely to be flown near maximum lift-to-drag ratio which means $C_{0}^{2}+S_{0}^{2} \approx 1$. This in itself severely restricts the possible values of $S_{0}$ and $C_{0}$. Further simplification results because the problem is reduced to determining three parameters as a result of the Hamiltonian integral (24).

There are ten equations to be integrated; namely eqns (11), (19), (20), (22) and (23). There are four initial parameters, $C_{0}, S_{0}, F_{0}$ and $G_{0}$, to be considered. The integration must satisfy the iransversality condition (31) and suitable terminal conditions for given initial conditions.

In the following, two types of optimal aerodynamic maneuver are considered. First, the one with the hard maneuver is studied. For this kind of maneuver, the vehicle will initially pull itself into the atmosphere, perform the skip maneuver with plane change, and subsequently exit to $h_{f}=h_{0}=0$, with a prescribed velocity depletion. The second kind of maneuver, which is studied next, is such that the vehicle stays at high altitude for the whole duration of the maneuver. Over each revolution, the motion is nearly Keplerian with small change in the energy, eccentricity and inclination during each passage through the perigee where the aerodynamic force is effective. The reason to study the single-pass maneuver is to show the usefulness of the set of equations of motion (11) we propose in this study. This kind of maneuver has been studied by Vinh and Hanson [4] using the modified Chapman variable $Z[5]$ as the dimensionless altitude. But the variable $Z$ is not suitable for the study of high altitude maneuvers, because in the numerical computation the $Z$ variable, which is essentially the dimensionless density of the atmosphere, may become negative when the vehicle reaches very high altitude near the apogee. It will be apparent by the end of this section that the new equations cover the whole range of altitude.

\subsection{Optimal plane change with single-pass maneuter}

It has been shown by Vinh et al. [2] that, for deep reentry trajectories. the initial entry altitude can be somewhat arbitrarily selected. Then, in the problem of the single-pass maneuver. the choice of $B=0.006$ does not affect the quantitative results. On the other hand, $\gamma$ is chosen to be a shallow angle, but the one which is steep enough to allow maneuverability in the atmosphere. By entering at 100 small an angle, the vehicle will exit the atmosphere without being able to do much maneuvering unless very high lift coefficients are used. On the other hand, too great an entry angle will be counterproductive because the vehicle will rapidly descend into the dense atmosphere and will lose velocity quickly. Hence the best effect is achieved by choosing the smallest $\%$ which allows effective maneuvers at reasonable values of lift coefficient The values of $\gamma_{0}$ found to be appropriate for this study are

$$
\begin{aligned}
& -\gamma_{0}=3.5, \text { for an entry speed } u_{0}-1.3 . \\
& -\gamma_{0}=4.0, \text { for an entry speed } u_{0}=1.6 .
\end{aligned}
$$


Table 1. Solutions of optimal single-pass maneuver

\begin{tabular}{lcc}
\hline$u_{0}$ & 1.3 & \multicolumn{1}{c}{1.6} \\
\hline$C_{0}$ & 0.1722642 & -0.3950000 \\
$S_{0}$ & 0.9294202 & 1.0146500 \\
$G_{0}$ & -0.1633919 & -0.1888347 \\
$F_{0}$ & 0.6499545 & -2.1182770 \\
$I_{\mathrm{f}}$ & $10.60^{\circ}$ & $19.61^{\circ}$ \\
\hline
\end{tabular}

The initial values

$$
\theta_{0}=\psi_{0}=\phi_{0}=\mathrm{h}_{0}=0
$$

are applied.

The initial values $C_{0}, S_{0}, F_{0}$ and $G_{0}$ must be chosen to satisfy the end conditions and from eqn (24) evaluated at the initial time as

$$
\begin{aligned}
& F_{0} \tan \gamma_{0}-\frac{B}{2 \cos \gamma_{0}}\left(1-S_{0}^{2}-C_{0}^{2}\right) \\
& \quad-\frac{E^{*} \tan \gamma_{0}}{u_{0}}+C_{0}\left(1-\frac{1}{u_{0}}\right)=0 .
\end{aligned}
$$

In this problem, the procedure will be to choose $C_{0}$, $S_{0}$, and $G_{0}$ and to solve for $F_{0}$. The final longitude $\theta_{\mathrm{f}}$ and the flight path angle $\gamma_{\mathrm{f}}$ are free. For a given final value of $u_{\mathrm{f}}=1.0, I_{\mathrm{f}}$ is to be maximized. Hence, the transversality condition (31) and in addition, the condition

$$
p_{\gamma_{\mathrm{f}}}=0 \text { or } C_{\mathrm{f}}=0
$$

must be satisfied. The integration will stop at

$$
h_{\mathrm{f}}=h_{0}=0 \text {. }
$$

The condition $\gamma_{\mathrm{f}}=0$ is very difficult to satisfy. This difficulty arises because the natural tendency of the trajectory is for $\gamma$ to increase when $u>1$. Since $\gamma>0$ for the ascending part of the trajectory, the vehicle must use a very high lift coefficient pointing downward to cause $\gamma_{\mathrm{f}}=0$. Therefore, $p_{\gamma_{\mathrm{f}}}=0$ was chosen as the better final condition. The solutions are shown in Table 1.

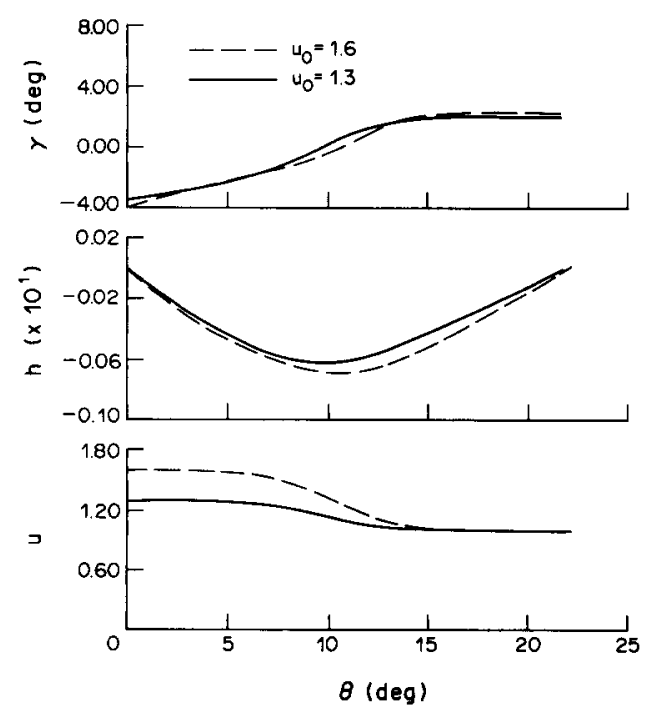

Fig. 2. The flight path angle, the altitude and the velocity vs the longitude (single-pass maneuver solution).
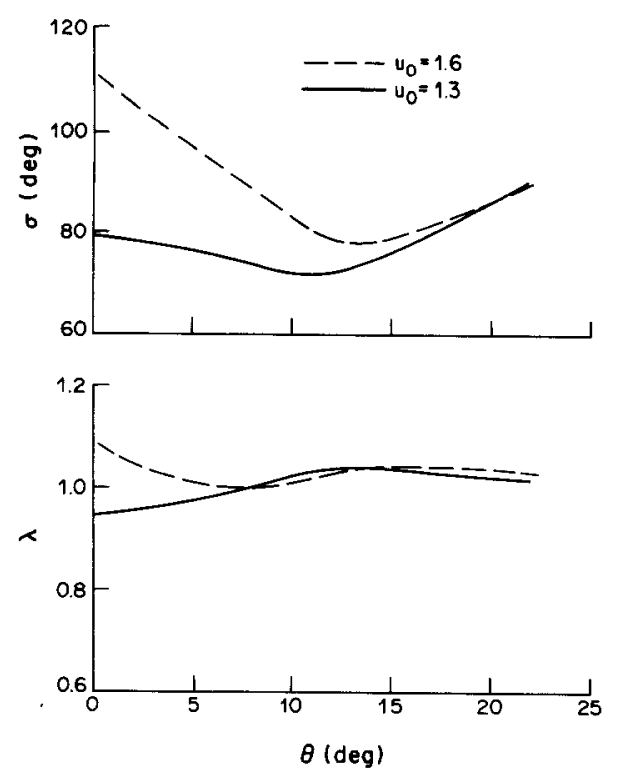

Fig. 3. The bank angle and the lift coefficient (single-pass maneuver solution).

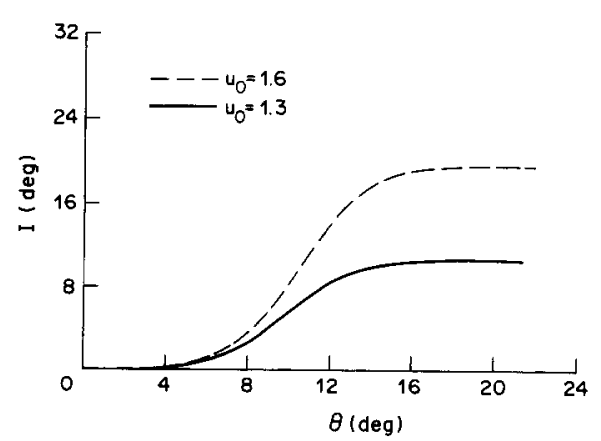

Fig. 4. The inclination angle vs the longitude (single-pass maneuver solution).

The variations of the flight path angle, altitude and speed for the two optimal skip trajectories are shown in Fig. 2. The variations of the bank angle and the normalized lift coefficient are shown in Fig. 3 while Fig. 4 displays the increase in the inclination.

\subsection{Optimal plane change for multiple-pass maneuver}

In this section the optimal trajectory for plane change of multiple-pass maneuver is studied. The equations to be integrated are the same as those used previously. The differences are the initial values of the four parameters $S_{0}, C_{0}, F_{0}$ and $G_{0}$ and the initial flight path angle $\gamma_{0}$ which is taken as the value of 0 to ensure that the vehicle will not fly into the atmosphere. The vehicle will stay in the orbit for several revolutions and accomplish the plane change at high altitude.

The value of the maximum lift-to-drag ratio to be used in this section is 1.5 , as used before. The planet is the Earth, and hence $\epsilon=1 / 900$. The value of $B$ to be used is $B=0.09$. It is low enough for the vehicle to stay in orbit for several revolutions. We also 
Table 2. Optimal solutions for multiple-pass maneuver

\begin{tabular}{lcc}
\hline$u_{0}$ & \multicolumn{1}{c}{1.3} & \multicolumn{1}{c}{1.6} \\
\hline$C_{0}$ & -0.0002692 & -0.00016567 \\
$S_{0}$ & 1.00069 & 1.00069 \\
$G_{0}$ & 0.000285 & 0.000285 \\
$F_{0}$ & 1.81683 & 1.7170 \\
$I_{\mathrm{f}}$ & $11.23^{\circ}$ & $20.13^{\circ}$ \\
\hline
\end{tabular}

consider two cases, $u_{0}=1.3$ and $u_{0}=1.6$. With the initial conditions, we have from eqn (24)

$$
-\frac{B}{2}\left(1-S_{0}^{2}-C_{0}^{2}\right)+C_{0}\left(1-\frac{1}{u_{0}}\right)=0 .
$$

This means that $C_{0}$ and $S_{0}$ are not independent of each other. Hence, instead of adjusting the parameters $C_{0}$,
$S_{0}$ and $G_{0}$ as has been done before, the parameters $F_{0}$, $S_{0}$ and $G_{0}$ must be given initially. The value of $C_{0}$ is obtained from eqn (37). Hence, $S_{0}$ is the only parameter with a physical meaning. This will increase the difficulty in solving the problems. The final longitude $\theta_{f}$ is free. The problem is that, as stated before, for a prescribed final velocity $u_{\mathrm{f}}=1.0, I_{\mathrm{f}}$ is to be maximized. Hence the transversality condition (31) must be satisfied. To ensure that, after the plane change is accomplished, the vehicle is still in orbit, the final flight path angle $\gamma_{\mathrm{f}}=0$ and the final altitude $h_{\mathrm{f}}=0$ must be enforced. In summary, we integrate the equations with the given initial values of the state variables and the selected parameters $S_{0}, F_{0}$ and $G_{0}$ and the computed value $C_{0}$. The integration will continue until
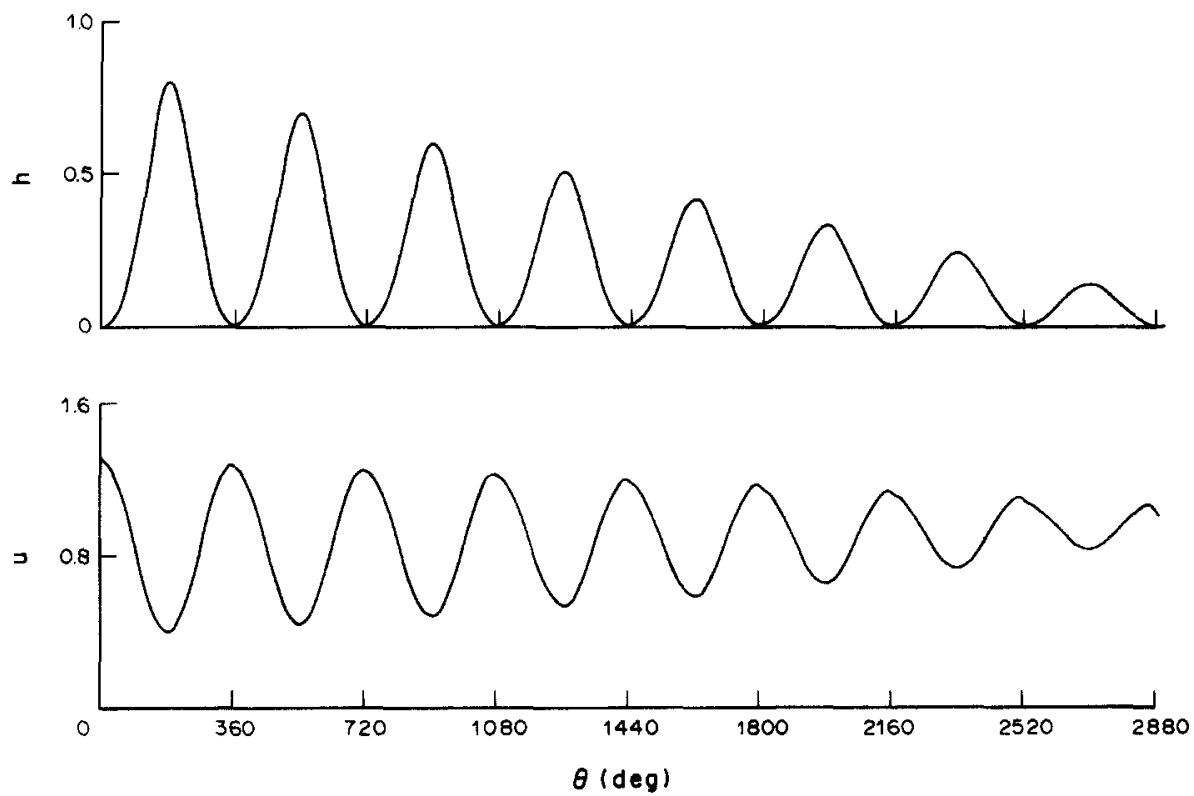

Fig. 5. The altitude and the velocity vs the longitude (optimal solution, $u_{0}=1.3$ ).
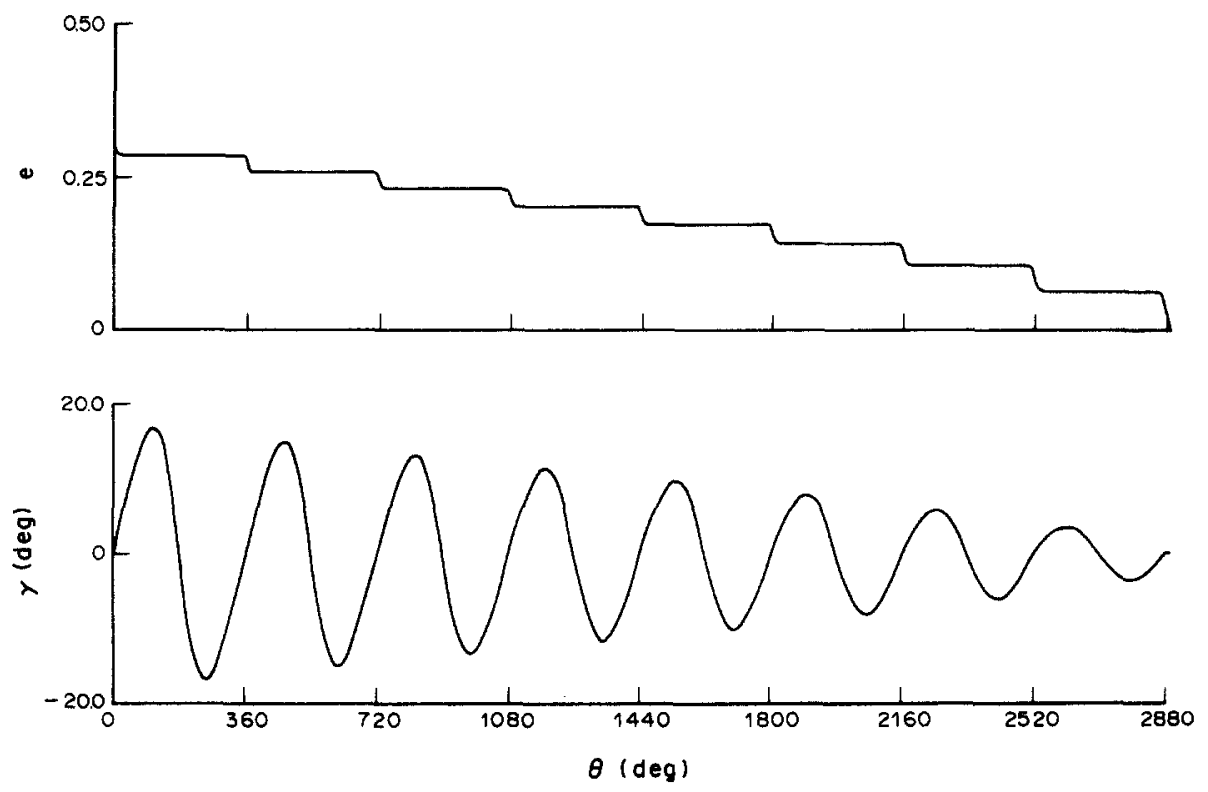

Fig. 6. The eccentricity and the flight path angle vs the longitude (optimal solution, $u_{0}=1.3$ ). 

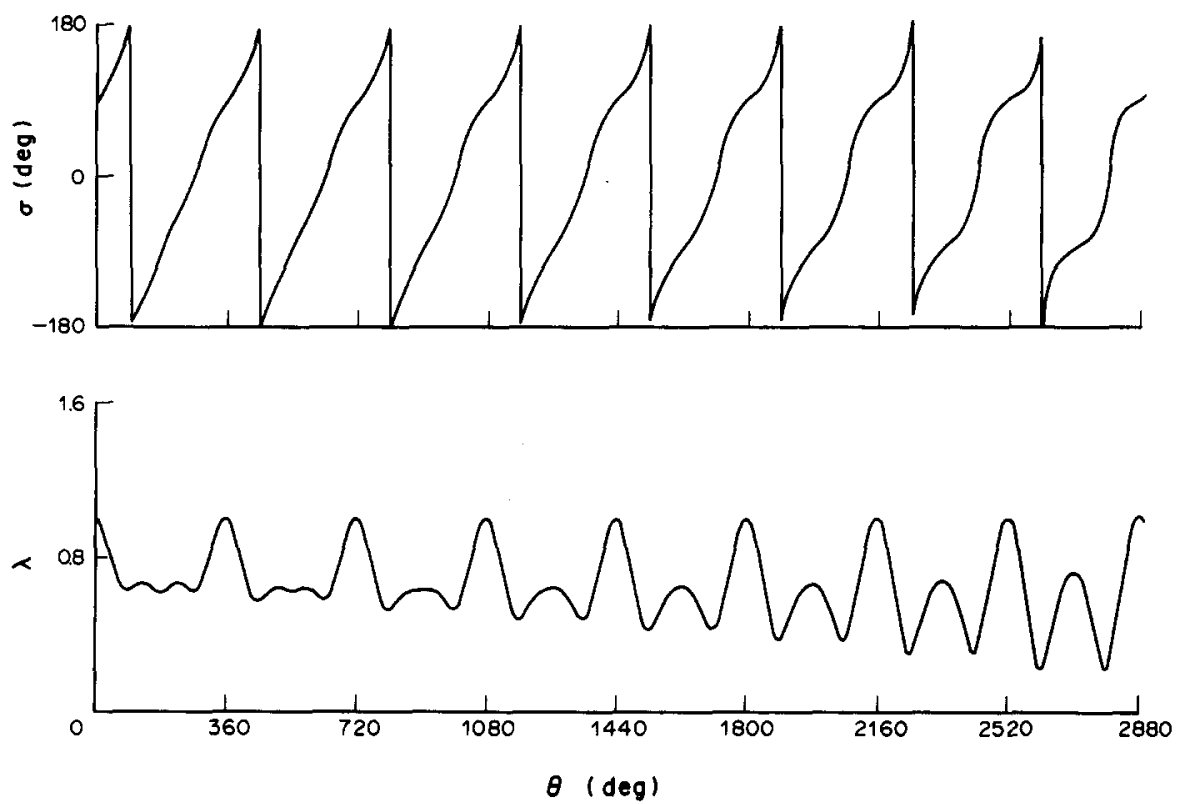

Fig. 7. The bank angle and the lift coefficient vs the longitude (optimal solution, $u_{0}=1.3$ ).
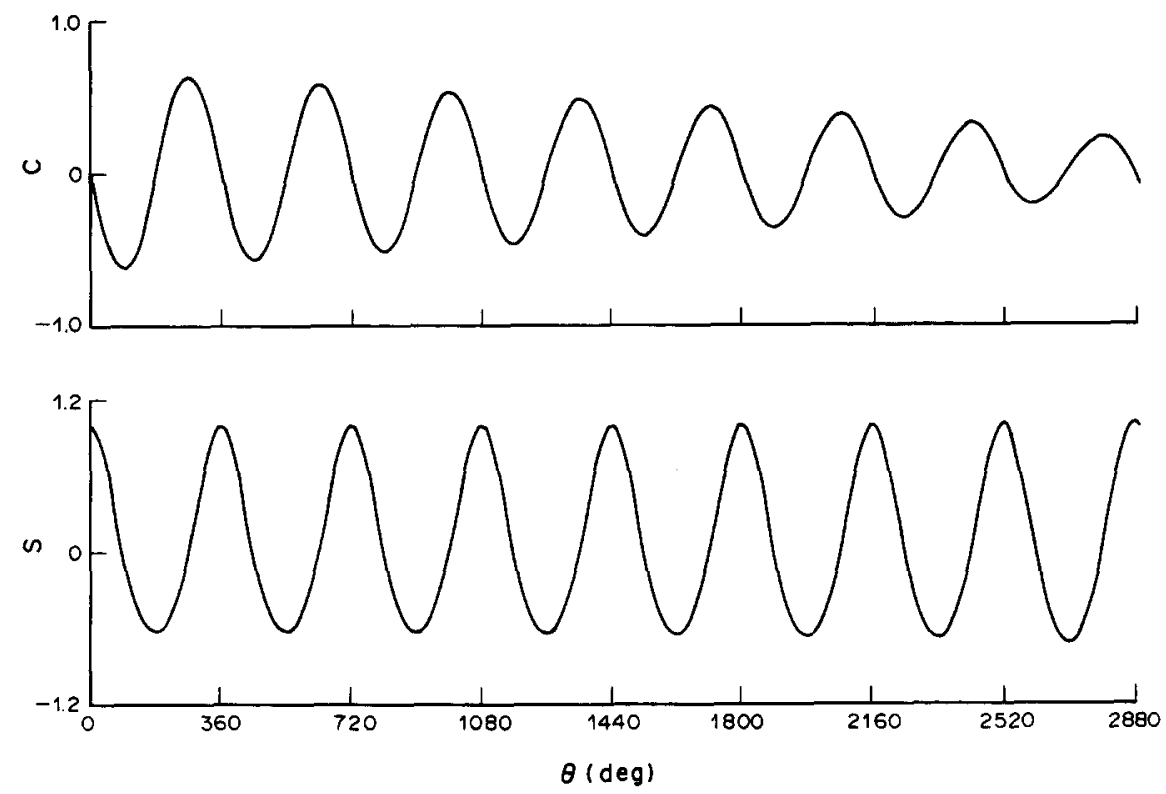

Fig. 8. The vertical and the lateral components of lift coefficient vs the longitude (optimal solution, $\left.u_{0}=1.3\right)$.

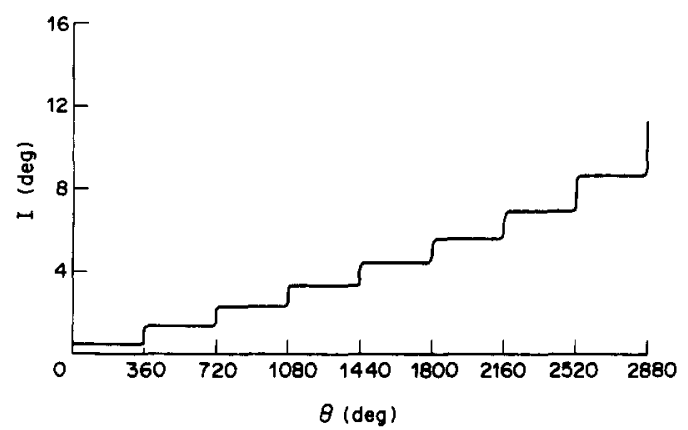

Fig. 9. The inclination angle vs the longitude (optimal solution, $u_{0}=1.3$ ). $u_{\mathrm{f}}=1.0$. Then the transversality condition (31) and the final conditions $\gamma_{\mathrm{f}}=0$ and $h_{\mathrm{f}}=0$ are checked for compliance. It can be shown through small flight path approximation for the first passage that $S_{0} \approx 1$, $F_{0} \approx E^{*}$. This restricts the range of parameter guessing and the solutions are shown in Table 2. These solutions are obtained with the accuracy of

$\left|\gamma_{\mathrm{f}}\right|, \quad\left|h_{\mathrm{f}}\right|, \quad\left|\tan \psi_{\mathrm{f}}+\sin \phi_{\mathrm{f}} \tan \left(\eta+\theta_{\mathrm{f}}\right)\right| \leqslant 0.001$.

To try to shrink the region is time consuming without much improvement in the plane change.

Figures 5-9 are the plots that show the variations of the state variables and the control along the 

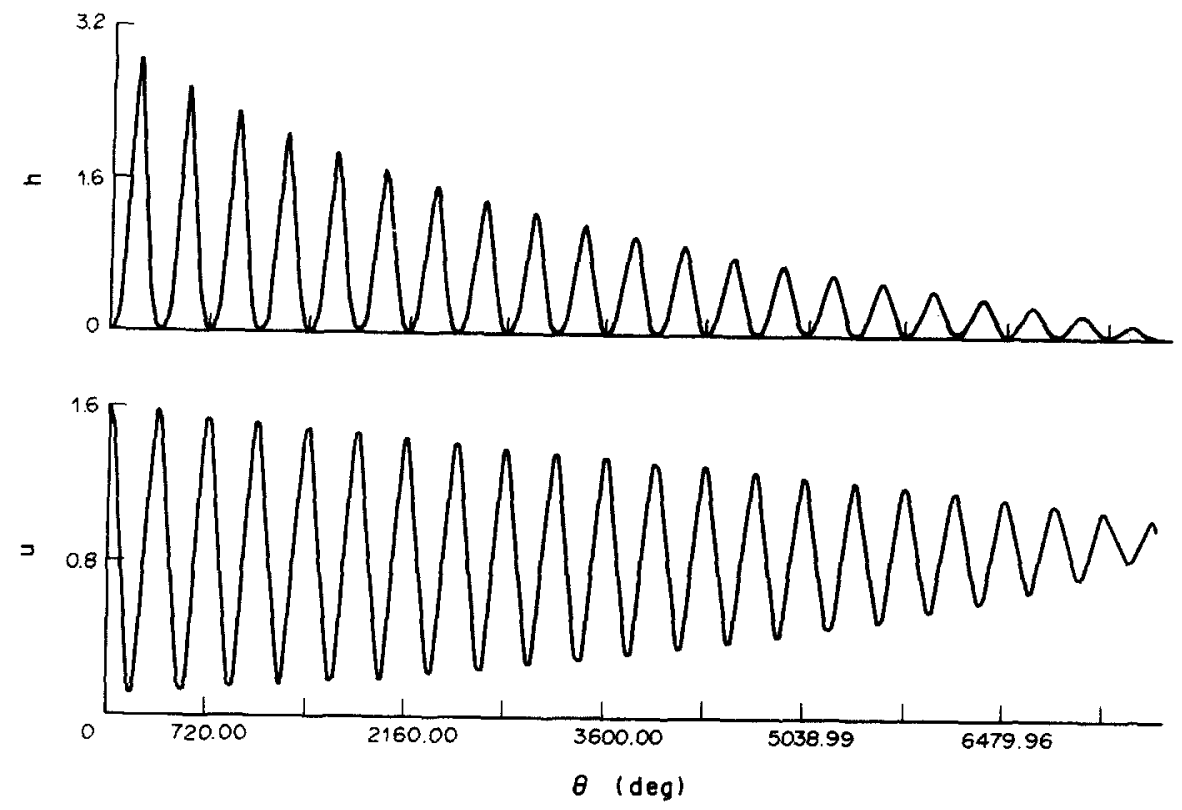

Fig. 10. The altitude and the velocity vs the longitude (optimal solution, $u_{0}=1.6$ ).

trajectory for $u_{0}=1.3$. For $u_{0}=1.6$, the plots are Figs 10-14.

By comparing the results from Tables 1 and 2, we see that the plane change for slow aerodynamic maneuver is nearly the same as for the case of hard maneuver. Hence plane change by multiple passages is of interest since it allows the vehicle to stay longer in orbit for other missions while it is obvious that slow motion incurs less heating protection.

The two sets of figures display similar behavior so that we discuss the case of high entry speed $u_{0}=1.6$ which corresponds to an initial eccentricity of $e_{0}=0.6$.
As shown in Fig. 10, the apogee altitude decreases after each passage while the perigee altitude remains constant to avoid deep entry. The speed is slightly depleted after each passage through the perigee.

Figure 11 shows the stepwise decreases of the eccentricity for each atmosphere passage. Also the flight path angle tends nearly periodically toward circularization.

In Fig. 12 are the plots of the bank angle $\sigma$ and the normalized lift coefficient $\lambda$ as computed from the values of $C$ and $S$ obtained through the numerical integration. By using a positive $\lambda$, a bank angle $|\sigma|$ in absolute value greater than $90^{\circ}$ means that the lift
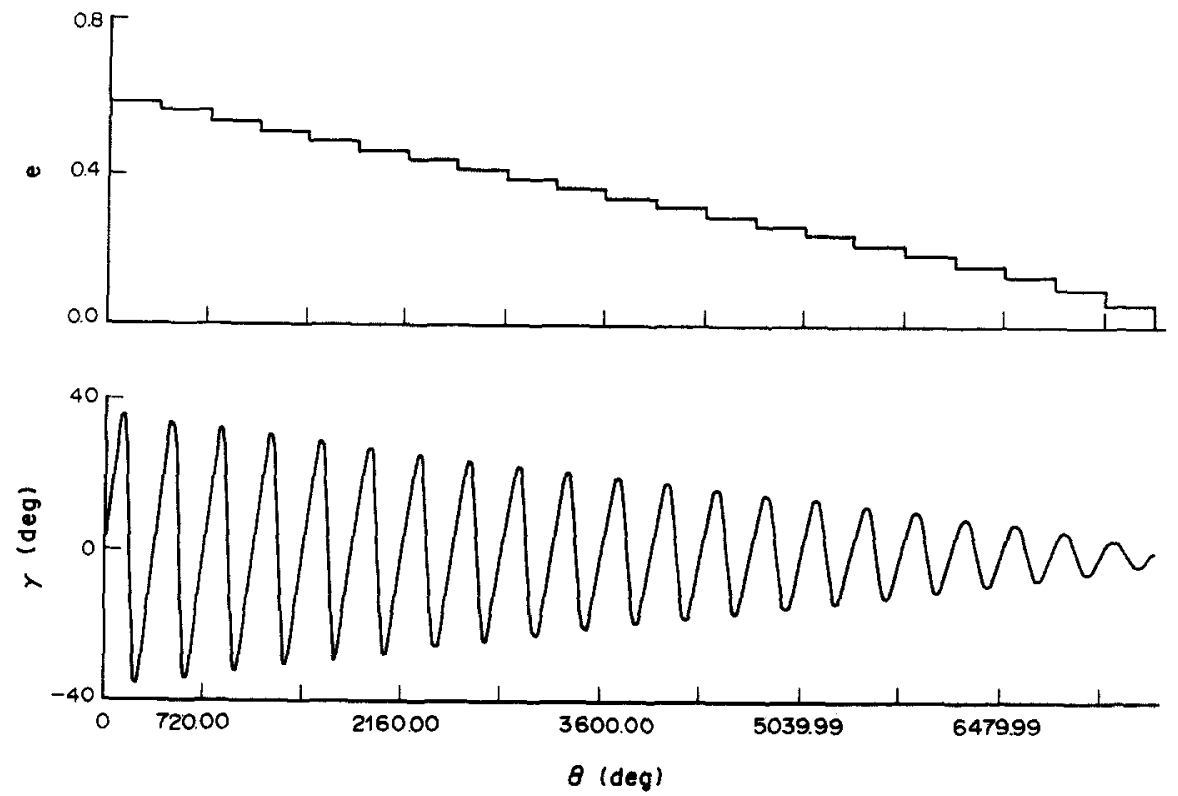

Fig. 11. The eccentricity and the flight path angle vs the longitude (optimal solution, $u_{0}=1.6$ ). 

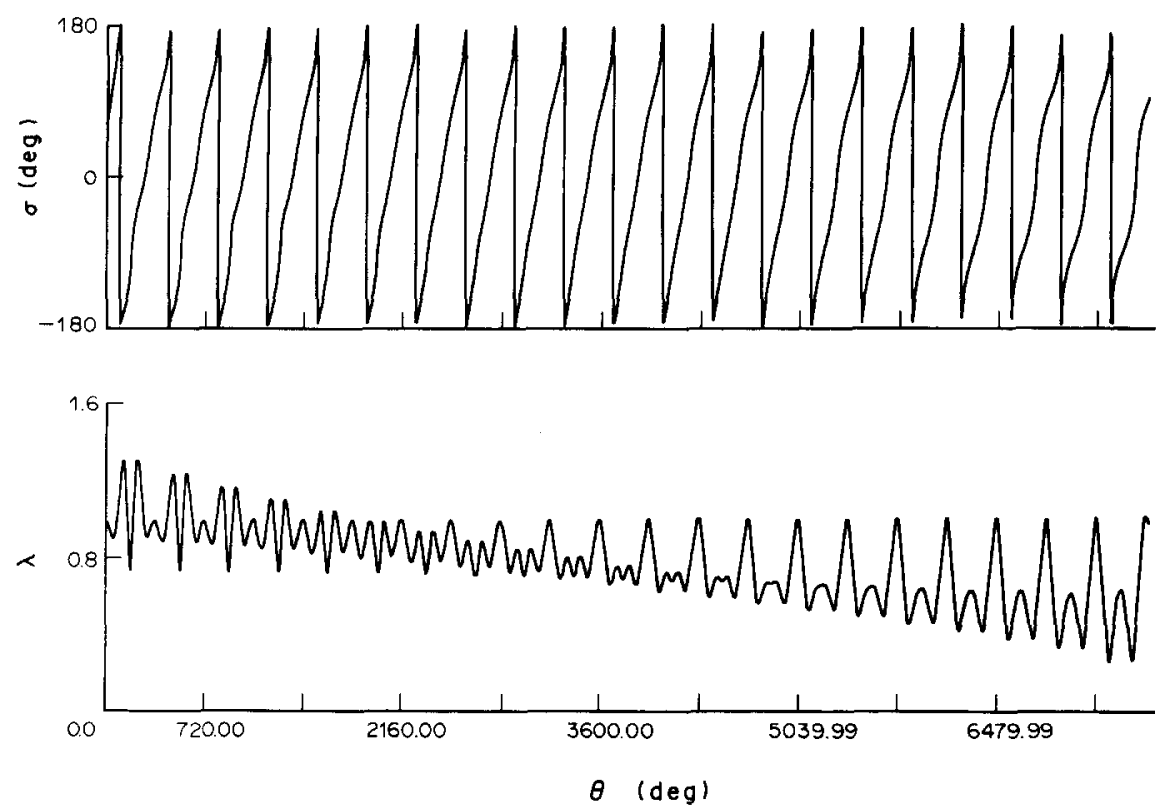

Fig. 12. The bank angle and the lift coefficient vs the longitude (optimal solution, $u_{0}=1.6$ ).

force is pointed downward. During each revolution the vehicle has a roll motion. The bank angle starts out near $90^{\circ}$ at the perigee and increases to overcome the tendency of the vehicle to skip out with positive flight path angle. It reaches $180^{\circ}$ at apex where the latitude is a maximum. It continues to roll and becomes $-180^{\circ}$ to be reduced at $-90^{\circ}$ at apogee. It is $0^{\circ}$ when the latitude is a minimum and continues to increase toward $90^{\circ}$ when the vehicle returns to the perigee.

Figure 13 shows the variations of the lateral component $S=\lambda \sin \sigma$ and the vertical component
$C=\lambda \cos \sigma$ of the normalized lift coefficient. Finally Fig. 14 shows the stepwise increase in the inclination.

Through the change from the trajectory variables used in this study to the orbital elements of the osculating orbit, it can be shown that the major axis decreases stepwise during the decay process while the longitude of the ascending node and the argument of the perigee remains nearly stationary. Hence while the orbit is contracting, it has a rotation stepwise about its line of apsides.
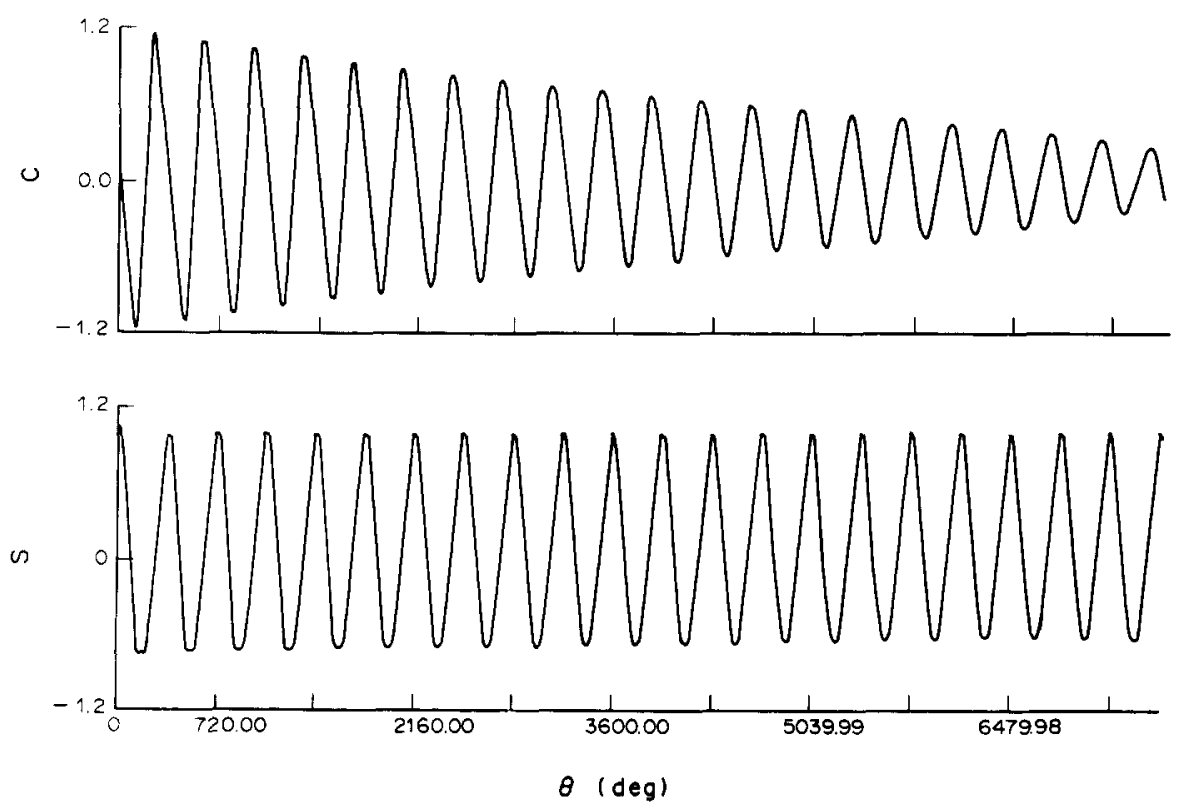

Fig. 13. The vertical and the lateral components of lift coefficient vs the longitude (optimal solution, $\left.u_{0}=1.6\right)$. 


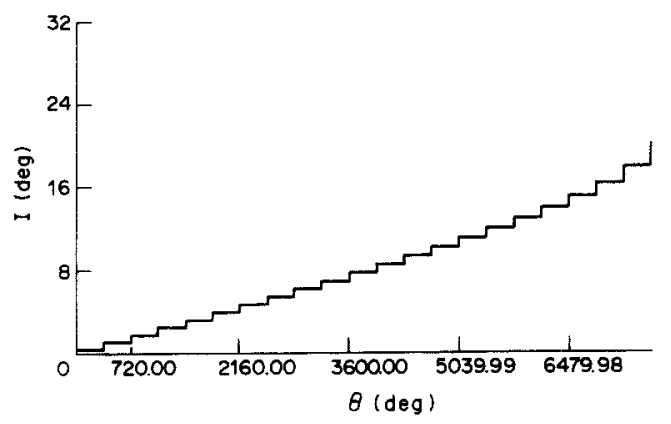

Fig. 14. The inclination angle vs the longitude (optimal solution, $u_{0}=1.6$ ).

\section{CONCLUSIONS}

In this paper, we have presented the exact dimensionless equations of motion of a lifting hypervelocity vehicle flying through the atmosphere of a spherical and non-rotating planet. The characteristic of the planetary atmosphere is specified by the parameter $\beta r_{0}$, taken as 900 for the Earth. The characteristics of the vehicle are specified by its maximum lift-to-drag ratio $E^{*}$, and the ballistic coefficient $B$. Only the value of $E^{*}$ has a definite influence on the performance which is here the maximum plane change for a prescribed energy loss.

The equations, and the associated variational equations obtained in the optimization process, can be used for the computation of the optimal atmospheric trajectories at orbital speed.

For the plane change, we can use the short duration maneuver with high deceleration or the long duration maneuver with low deceleration and heat rate with essentially the same resulting plane change.

Acknowledgement-This work was supported by the Jet Propulsion Laboratory under contract No. 956416 with Dr L. J. Wood as project manager.

\section{REFERENCES}

I. K. D. Mease, Optimization of aeroassisted orbit transfer; current status. $J A S$ 36, 7-33 (1988).

2. N. X. Vinh, A. Busemann and R. D. Culp, Hypersonic and Planetary Entry Flight Mechanics. University of Michigan Press, Ann Arbor (1980)

3. N. X. Vinh, Optimal Trajectories in Atmospheric Flight. Elsevier, Amsterdam (1981).

4. N. X. Vinh and J, M. Hanson, Optimal aeroassisted return from high Earth orbit with plane change. Acta Astronautica 12, 11-25 (1985).

5. J. S. Chern and N. X. Vinh, Optimal reentry trajectories of a lifting vehicle. NASA CR-3236 (1980). 